\title{
Towards a Domain Specific Modeling Framework for General Collective Intelligence Platforms
}

\author{
Andy E. Williams, Nobeah Foundation, Nairobi, Kenya
}

\begin{abstract}
:
A General Collective Intelligence or GCI is a hypothetical platform able to self-organize individuals into potentially massive networks of cooperation on a self-sustaining basis, where those networks might radically increase the group's general problem-solving ability and hence ability to solve any problem in general, which translates to a radical increase in the capacity of the group to achieve any of it's collective goals. This potential to significantly increase collective outcomes has been confirmed through analysis of GCI based platform designs targeting social good, such as a proposed "Social Impact Marketplace". In software, GCI is predicted to create the potential to enable social media platforms, blockchain platforms, or any other type of platform, to self-assemble at a speed, scale, and size of user base not currently possible, while also providing functionality that reliably solves problems that are currently not reliably solvable. In order to achieve this, GCI leverages the concept of "functional state spaces" to provide a semantic representation of the functionality in each domain of software behavior. This paper explores the concept of functional state spaces as a framework for domain specific modeling, as well as how such functional state spaces potentially provide a semantic representation of the functionality in each domain that is universal and therefore suitable for a General Collective Intelligence platform capable of targeting any given problem in any given domain.
\end{abstract}

\section{Keywords}

domain specific modeling; General Collective Intelligence, Human-Centric Functional Modeling; functional state space; conceptual space

\section{Introduction}

Where the generation of implementations of models is possible (model-driven engineering), particularly in the case of software, it is well known that defining models representing the behavior of systems in any given domain (domain specific modeling) can radically accelerate the development process. However, domain specific modeling as currently implemented has a number of shortcomings, some of which are:

- Models might not represent the complete set of behaviors of a system in a given domain. Implementations of any modeling elements that are used in domain specific tools to generate implementations of models might be a small subset of the possible implementations and might not be optimal in all circumstances.

- When the number of operations represented in a domain specific modeling language becomes large the models become unwieldy and potentially unusable for humans.

- Applying expert knowledge like software architecture patterns is a time-intensive manual process.

This paper explores how functional state spaces might be used to define complete models of the behavior of systems in a given domain, and how domain specific models defined this way might leverage General Collective Intelligence to radically increase the number of elements in such models that might be manageable for a human, as well as to radically increase the speed and scale of systems development.

A General Collective Intelligence or GCI [1] is a hypothetical platform able to self-organize individuals into potentially massive networks of cooperation on a self-sustaining basis, where those networks might exponentially increase the general problem-solving ability (collective intelligence) of the group. 
Though a complete GCI has not yet been implemented to demonstrate general problem-solving ability across all domains, a sufficient subset of GCI has been approximated in the design of various platforms to confirm this potential to exponentially increase problem-solving ability within specific domains [2].

Functional state spaces are graphs of networks capable of representing the complete behavior of systems. In these graphs each node represents a functional state of the system, and each edge represents a behavior through which the system might transition from one functional state to another. A GCI contemplates systems within its collective reasoning processes for the purposes of collectively solving problems concerning those systems. For example, a GCI might contemplate a model of each blockchain platform as a system, in order to solve problems concerning security, scalability, or decentralization in such platforms. Within a GCI, information (both concepts and reasoning) about each system contemplated by collective reasoning is represented in terms of a collective space of concepts or "collective conceptual space" by analogy with the individual conceptual space that serves as the functional state space of the human cognitive system. In essence, a GCI might model the behavior of the systems themselves, where each system model can be represented as having behaviors contained within its own functional state space, and as the system exhibits those behaviors it navigates that space. But since cognition is itself a behavior of a system (the cognitive system), GCI as a collective cognition might model thoughts (information) about systems, which are represented within the collective conceptual space. So as a system, a blockchain or other platform might have a representation in the collective conceptual space of the GCI, or it might have a representation in the functional state space of the blockchain or other platform itself. Because these representations can be different, the optimal representation must be selected [15]. Problem-solving ability is predicted to be optimized when modeling system behavior in terms of the functional state space of the system since such models only represent actual behaviors rather than theorized behaviors that might not actually exist, but because such models are difficult to define and therefore themselves might not exist, the only option might be to model information about systems in the functional state space of the cognition. The validity of any representation of the blockchain platform in the conceptual space is determined by whether that representation is a valid semantic model. The validity of any representation of the blockchain platform in a hypothetical functional state space describing all blockchain platforms is determined by whether that model is valid in the semantics of that representation. In either case, both functional state spaces define complete representations for their respective systems (the cognitive system and the blockchain or other platform), and in the sense that a semantic representation is a complete description of meaning, both are semantic representations. The difference is that one (the description in conceptual space) defines the semantics of human-meaning. The other defines the semantics of the system itself in a human-centric way through the technique of Human-Centric Functional Modeling. This paper explores this underlying technique of Human-Centric Functional Modeling and the functional state spaces it defines as a general methodology for representing systems within each domain of behavior and as the basis for domain specific modeling.

\section{Domain Specific Modeling and Functional State Spaces}

Human-Centric Functional Modeling or HCFM represents systems as having a set of processes with which the system might transition from one state to another within each domain of system behavior. These processes are defined as being within the same domain of behavior if their input and output belong to the same category shared by all processes within that domain. That is, if their input and output consist of the same domain object. These processes can potentially all be represented in terms of some minimal set of basic functions, in which case the set of those basic functions can be said to "span" all such processes. These states are then referred to as "functional states" because they are defined solely in terms of those functions. All of these states together form a space of functional states or a "functional state space" that the system moves through. 
As an example, in the domain of cognition, the human cognitive system can be represented as having an open set of reasoning processes (open in the sense that reasoning processes can be added or removed). Such processes are represented as belonging to the domain of cognition because both their inputs and outputs are all concepts, where the concept is the domain object. The resulting space of concepts or "conceptual space" forms the functional state space of the cognitive system. As the cognitive system engages in reasoning activity it changes concepts, and since the current concept defines its functional state, it therefore changes state, and in doing so it moves through its functional state space (the conceptual space). Furthermore, it is hypothesized that all paths in this conceptual space, and therefore all reasoning (all behaviors of the cognitive system), can be represented in terms of four basic functions [7]. If so, the set of these four functions can in this sense be said to "span" this conceptual space.

The concept of state spaces as semantic models and the concept of collective intelligence have been well explored in other disciplines. In cybernetics, the concept of a noosphere similar to the concept of a functional state space, and the concept of a global brain similar to the model of General Collective Intelligence (GCI) discussed in this paper, have been described by other authors [3]. In philosophy, there is an established tradition of using semantic models for representing information [4]. Bas C. van Fraassen has been attributed credit [5] for the concept of the "state space" as a semantic modeling approach, and a number of researchers citing his work have taken the concept of "state spaces" and applied them to all of science in general, including to physics and to modeling the physical world [6].

Within software development, constructing a functional state space for a given domain of platform level architecture services requires defining a basic set of functions that "spans" all possible behavior by all possible systems within that category. As an example, as mentioned, it is hypothesized that all possible reasoning or understanding processes within the human cognitive system can be composed by four basic functions [7]. Furthermore, since these functions can span the entire conceptual space, and since this conceptual space is believed to be identical to any other open (unbounded) functional state space, then it is hypothesized that any other open functional state space can potentially be spanned by four operations as well. A set of four such operations has been proposed for a hypothetical blockchain domain [8], so that every set of functionality from every blockchain platform might be decomposed into a library of functions that a GCI based blockchain platform might use to enable interoperability between all platforms, as well as significantly improved performance over all other possible platforms, by intelligently navigating an exponentially greater number of choices to select the functionality most suited to achieve its targeted outcome in each specific situation.

However, a set of processes or functions does not need to "span" an entire domain to be useful. It might instead describe some useful subset of that domain. That subset does not even need to be well-defined in order to be useful. It might be that a set of functions or processes are subjectively judged as describing a "useful" subset of a given domain. This subset of processes might be represented by some finite ontology. Ontologies however are notorious for becoming fractured into different incompatible versions. Efforts to define the next new ontology that captures "everything" have failed for as long as such efforts have existed. In the same way that efforts to define a complete English dictionary continued to fail until language was recognized to be a living entity with a description that must be ever evolving and can never be complete, the key problem might be the idea that the ontology is the most important information being captured, rather than the goal being capturing the processes by which ontologies or any other system of meaning evolves. 
An example of such an ontology is the eXtensible Domain Modeling Framework (XDMF), and an example of such a process for evolving that ontology is what has been described in Human-Centric Functional Modeling as "functional domain decomposition", which in HCFM is hypothesized to be an an internal (unconscious) function required for general problem-solving ability in any domain. This function is believed to allow nature to adapt to determine which functionality will be within one given system as opposed to another, and to allow nature to determine which functional components that functionality will be spread across, as well as what functionality will reside in each function. Deployed as an algorithm within a GCI platform acting as the collective cognition of a group, with such domain decomposition functionality it is hypothesized that groups might reliably converge on agreement about what functionality should be included in each domain, and which functional components each domain should be partitioned into when defining domain specific models, in order to maximize fitness in achieving outcomes that might be attained through executing any of the functions within that domain.

XDMF consists of a hierarchy of subject areas that are assumed to represent domains. Each domain consists of a number of operations that describe in plain natural language (in this case English) what kinds of actions can be done within that subject area. The only constraint placed on operations is that all operations in a single domain must act on the same type of object, the domain object.

Different nuances of the action described by a particular operation might in turn be described by a subdomain. The sub-domain contains a number of operations that all fall within the same category as the parent operation. The hierarchy of domains and sub-domains may be several layers deep. Part of the potential hierarchy of operations is shown in table 1 .

\begin{tabular}{|c|c|c|c|c|c|}
\hline Domain & $\begin{array}{l}\text { Domain } \\
\text { Object }\end{array}$ & $\begin{array}{l}\text { Domain } \\
\text { Operation }\end{array}$ & $\begin{array}{l}\text { Child } \\
\text { Domain }\end{array}$ & $\begin{array}{l}\text { Child } \\
\text { Domain } \\
\text { Object }\end{array}$ & $\begin{array}{l}\text { Child Domain } \\
\text { Operation }\end{array}$ \\
\hline \multirow{20}{*}{$\begin{array}{l}\text { Overall } \\
\text { Business } \\
\text { Operations } \\
\text { Domain }\end{array}$} & \multirow[t]{20}{*}{ Assets } & \multirow[t]{4}{*}{ ENGINEER } & \multirow[t]{4}{*}{$\begin{array}{l}\text { Engineering } \\
\text { Domain }\end{array}$} & \multirow[t]{4}{*}{ Products } & DESIGN \\
\hline & & & & & PROTOTYPE \\
\hline & & & & & BUILD \\
\hline & & & & & TEST \\
\hline & & \multirow[t]{16}{*}{ MANAGE } & \multirow{4}{*}{$\begin{array}{l}\text { Corporate } \\
\text { Administration } \\
\text { Domain }\end{array}$} & \multirow{4}{*}{ Policies } & SET \\
\hline & & & & & ENFORCE \\
\hline & & & & & REVIEW \\
\hline & & & & & REVOKE \\
\hline & & & \multirow{7}{*}{$\begin{array}{l}\text { Finance } \\
\text { Domain }\end{array}$} & \multirow[t]{7}{*}{ Finances } & FORECAST \\
\hline & & & & & BUDGET \\
\hline & & & & & ACCOUNT \\
\hline & & & & & COLLECT \\
\hline & & & & & STRATEGIZE \\
\hline & & & & & INVEST \\
\hline & & & & & ANALYZE \\
\hline & & & \multirow{4}{*}{$\begin{array}{l}\text { Human } \\
\text { Resources } \\
\text { Domain }\end{array}$} & \multirow{4}{*}{$\begin{array}{l}\text { Human } \\
\text { Resources }\end{array}$} & RECRUIT \\
\hline & & & & & HIRE \\
\hline & & & & & TERMINATE \\
\hline & & & & & SET POLICIES FOR \\
\hline & & & Information & Information & INTEGRATE \\
\hline
\end{tabular}




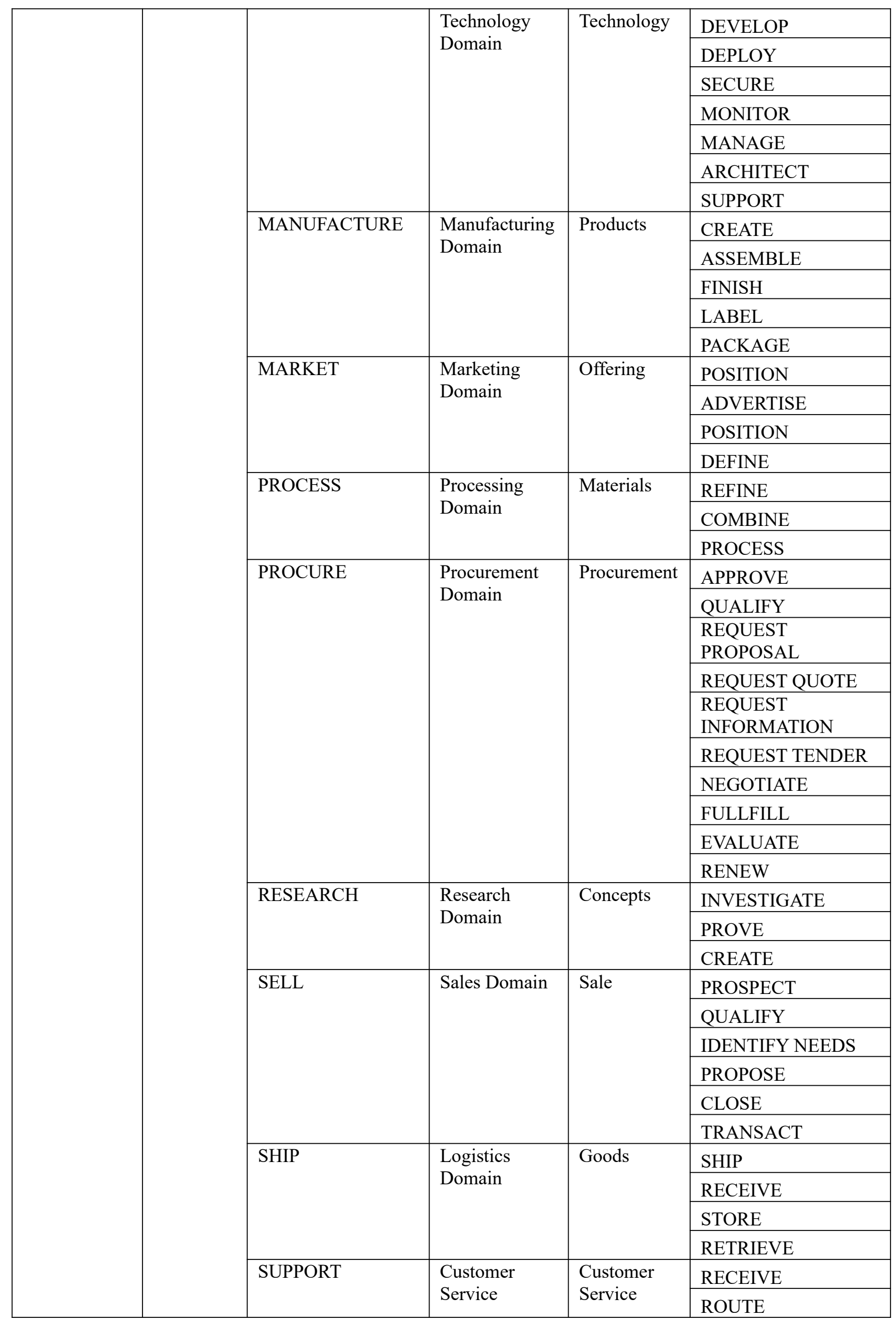




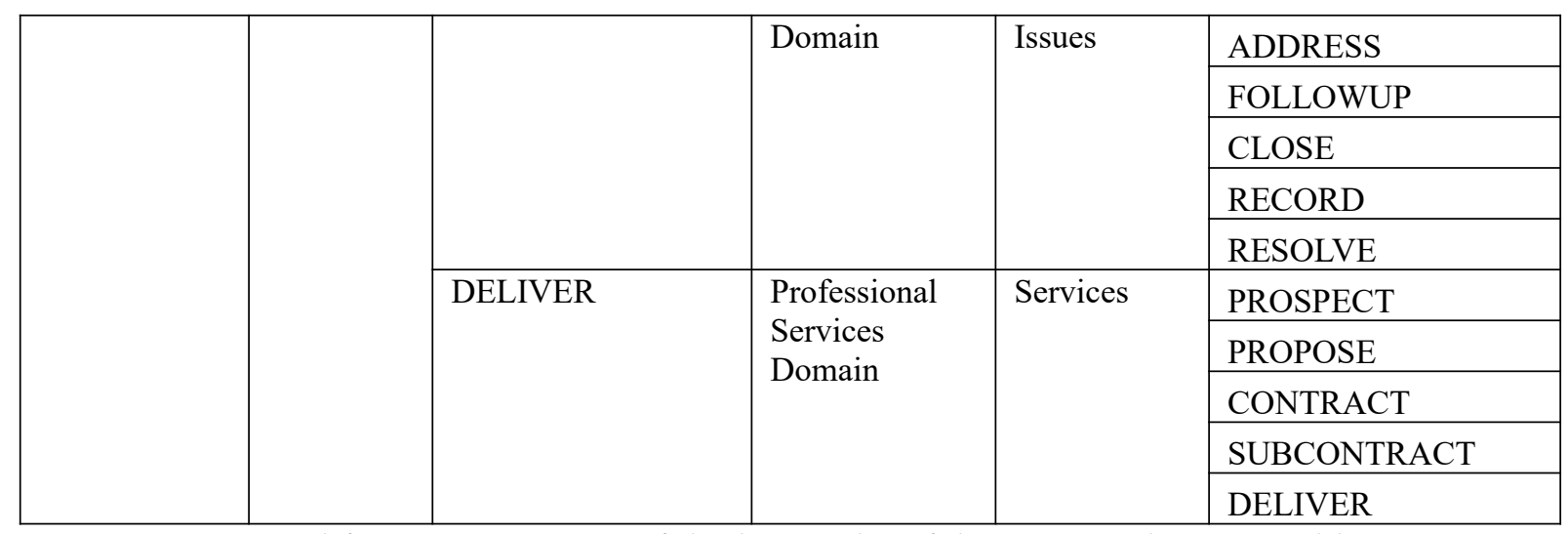

Table 1: Proposed functions in some of the hierarchy of domains in the eXtensible Domain Modeling Framework (XDMF).

This hierarchy of domains corresponds to a hierarchy of regions in the functional state space of the cognitive system as in figure 1 .

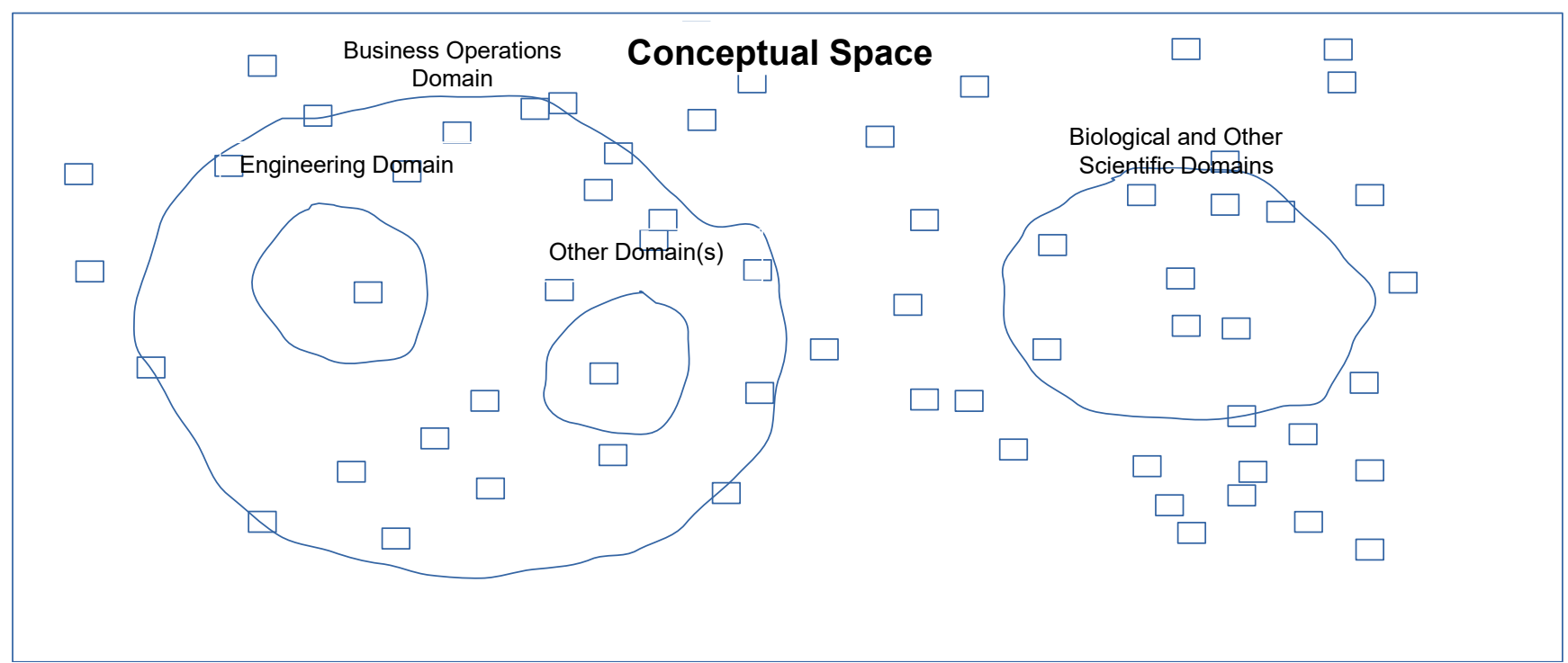

Figure 1: Functional state space and the corresponding hierarchy of domain specific models.

Again, the most important takeaway is not the particular set of domains proposed in table 1, or even the ontology of functions proposed. Instead the most important point is defining a process by which the fitness of a given ontology in achieving its targeted outcomes can be assessed, where this process might also be used to determine the fitness of the combination of all ontologies together, so that any component of any ontology, or even the entire ontology, or set of ontologies itself, might be replaced by one that is more fit. This metric of fitness applies to both to the fitness of the functional model in representing observed behavior, and the fitness of all implementations in terms of their performance in achieving targeted outcomes as shown in figure 2 . 


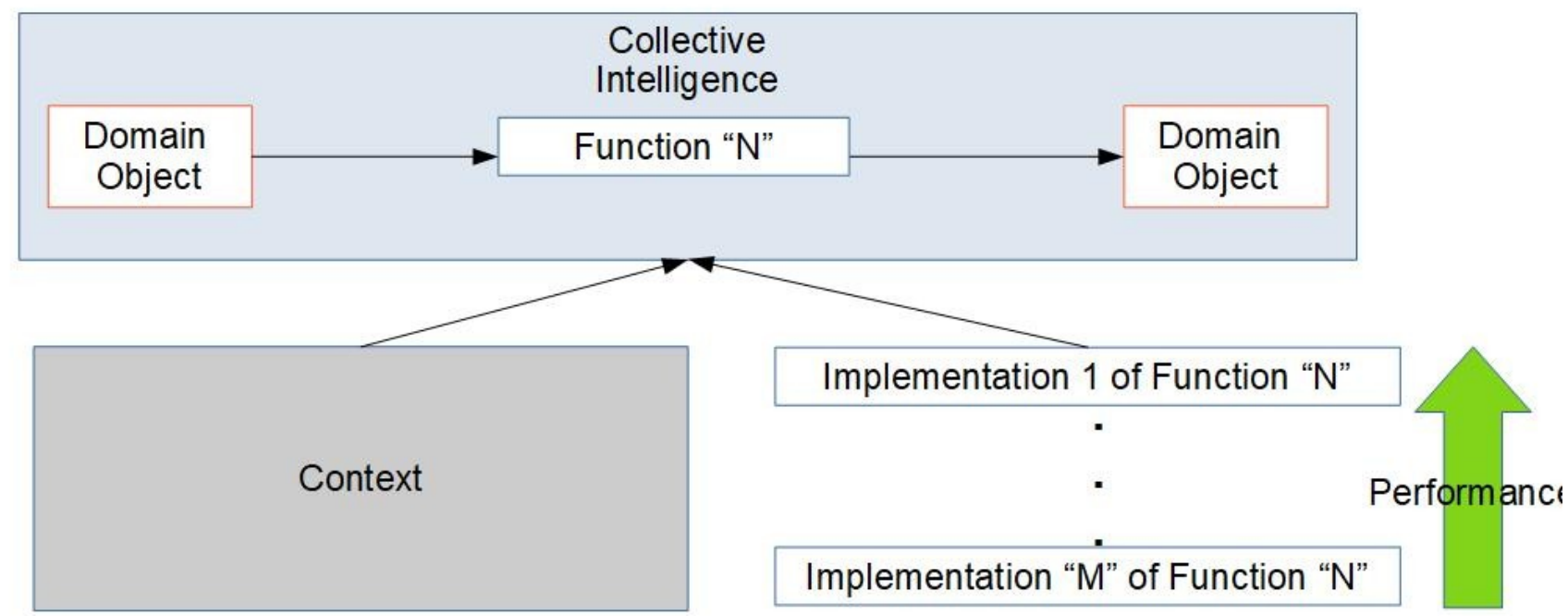

Figure 2: The collective intelligence uses uses a metric of fitness to select the optimal implementation of each function for the given context in order to increase projected performance.

In this way, the process of evolving ontologies becomes decoupled from any ontology itself. This decoupling is a hallmark of the Human-Centric Functional Modeling process itself, in which any functional component might be replaced by one that is more fit. Because of this attribute, it is hypothesized that while models of any system represented in HCFM might be wrong in terms of not representing valid behaviors of the system, HCFM itself cannot be wrong, because any component of it that doesn't exhibit fitness in representing observed behavior of any system it is being used to describe, can simply be replaced by a component that is more fit.

As mentioned, the operations within any given domain are confined to a single domain object as their arguments and as their output. As a whole, this framework of domains is called eXtensible because it is not intended to be definitive. It is intended to be flexible enough to expand or contract to suit different businesses, including new domains that arise as businesses continue to evolve. The important benefit that is constant is that the framework provides a complete way to model parts of businesses, as well as a consistent way to understand the impact of one modeled part of a business, on another modeled part of a business. 


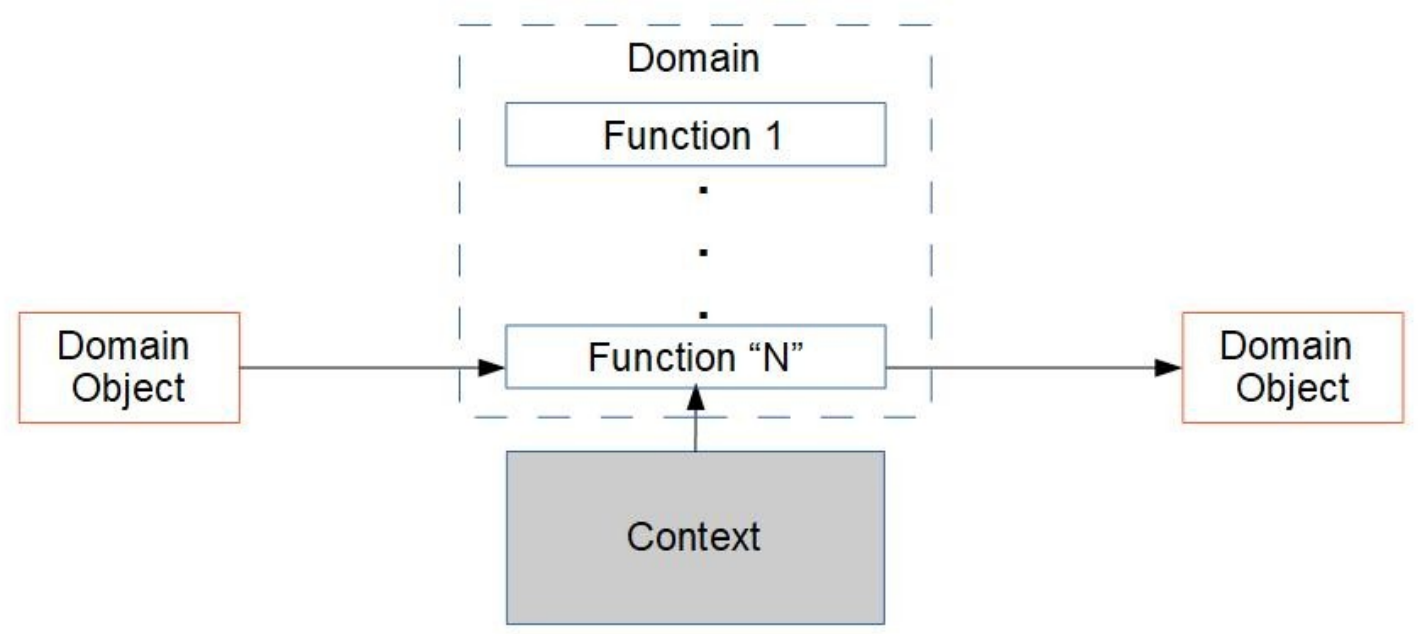

Figure 3: In the eXtensible Domain Modeling Framework (XDMF) each process or function receives a domain object as its input and produces that same type of domain object as its output. All functions in the domain then map the domain object back into the domain. The "context" of the function's execution are any additional domain objects of that same type, other than inputs or outputs, which help define the execution of those processes or functions.

Any one domain described by one ontology might also interact with another domain described by another ontology. In XDMF this interaction is represented as occurring through the exchange of domain objects. For this to be possible these domain objects themselves must have well-defined relationships. In order to ensure that definitions remain "human-centric" in terms of not requiring any special jargon, these definitions are intended to be based on the plain natural language meaning of the object names. Examples of domains that interact are the proposed Information Processing Domain Modeling Language for representing the majority of operations that can be executed on the text data that business logic largely manipulates, and the proposed Visual Processing Domain Modeling Language for representing the majority of operations that can be executed within any user interface.

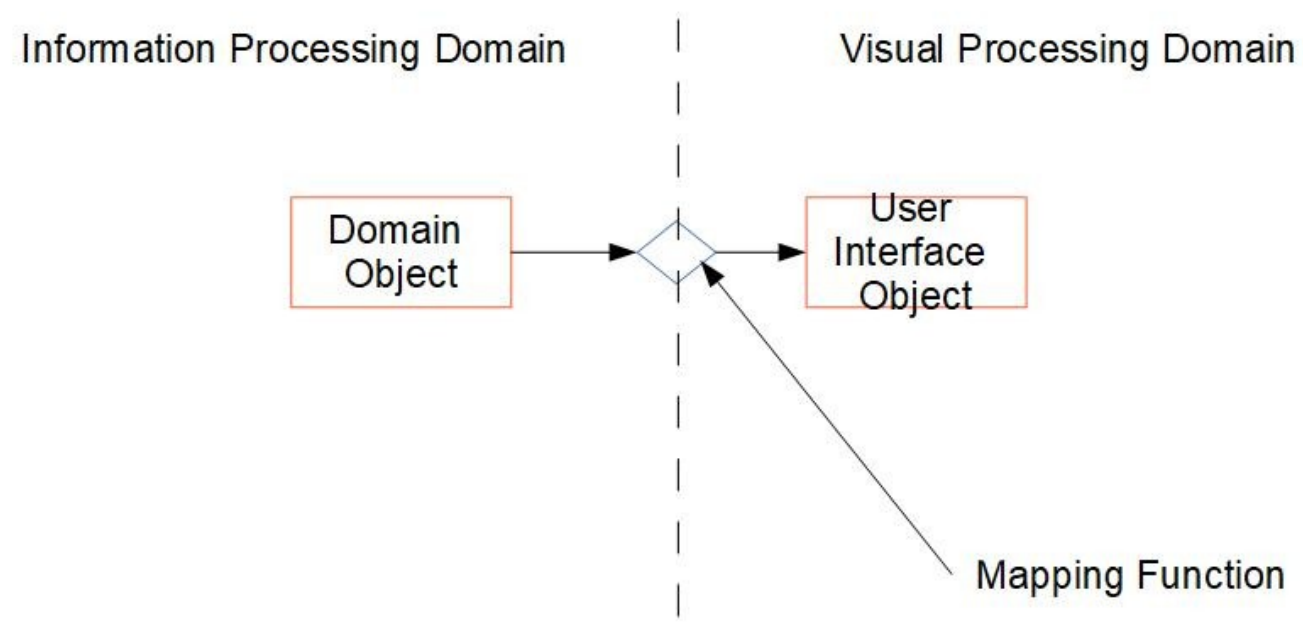


Figure 4: In domain mapping, a mapping function maps a domain object in one domain to a domain object in another. An example is the mapping of the proposed Information Processing Domain (IPD) to the proposed Visual Processing Domain (VPD).

A domain object representing a "customer" in the IPD requires only customer information. A domain object representing the display, or editing of that data in a user interface within the Visual Processing Domain specifies data about the widget with which each field in the customer data is best displayed within a given form factor of device, and specifies data about how those widgets should be displayed.

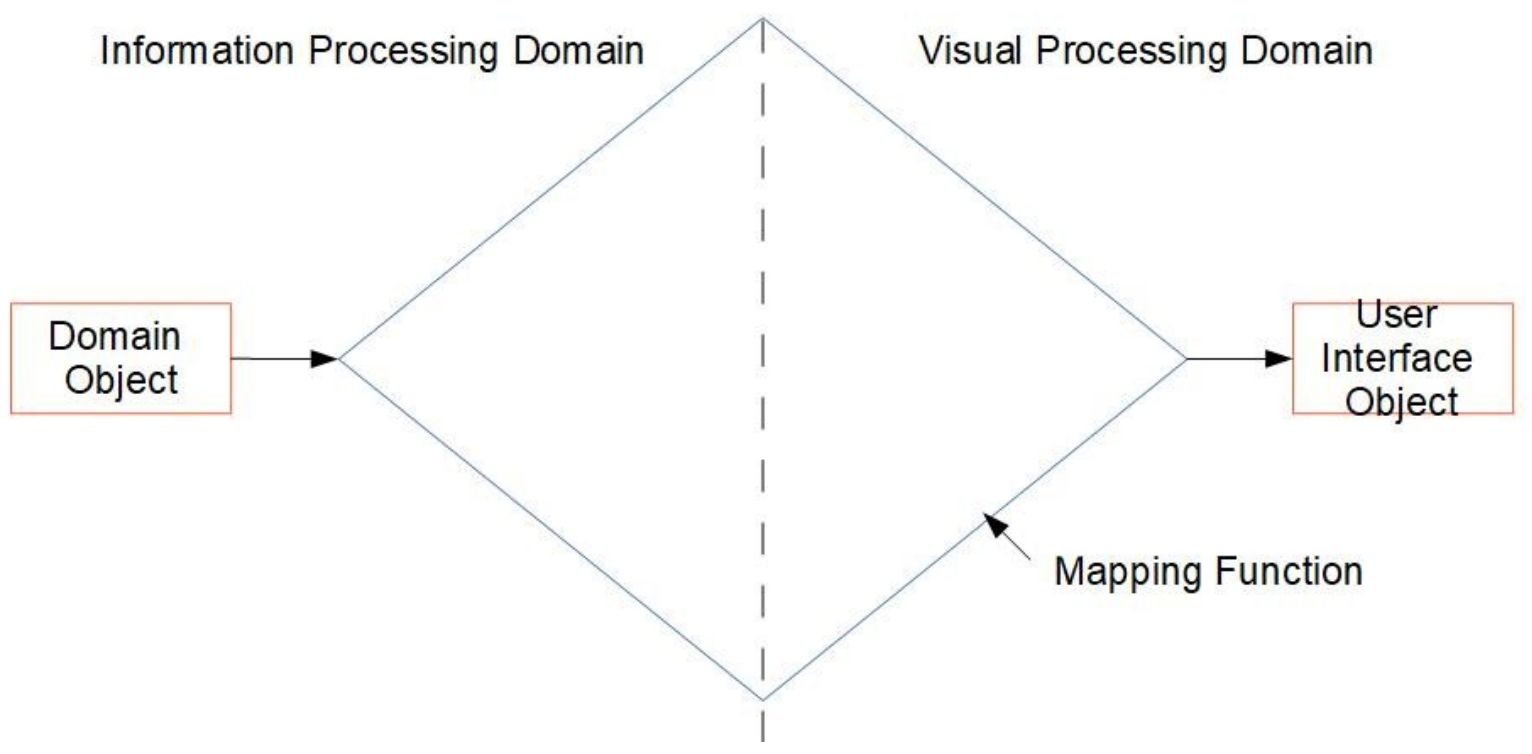

Figure 5: A domain object representing a "customer" in the IPD might be mapped to a user interface that collects customer data in the VPD.

By creating functional models for each component, we can enable each component to be used far more pervasively. By creating functional models for architecture level and other services involved in creating a platform for decentralized applications that can make use of such components in this way, and by defining metrics of fitness that allow us to compare these components at exponentially greater speed and scale, we can enable existing offerings that can be decoupled into such components to be selfassembled by their vendors into platforms far more quickly than any single company could build them. 


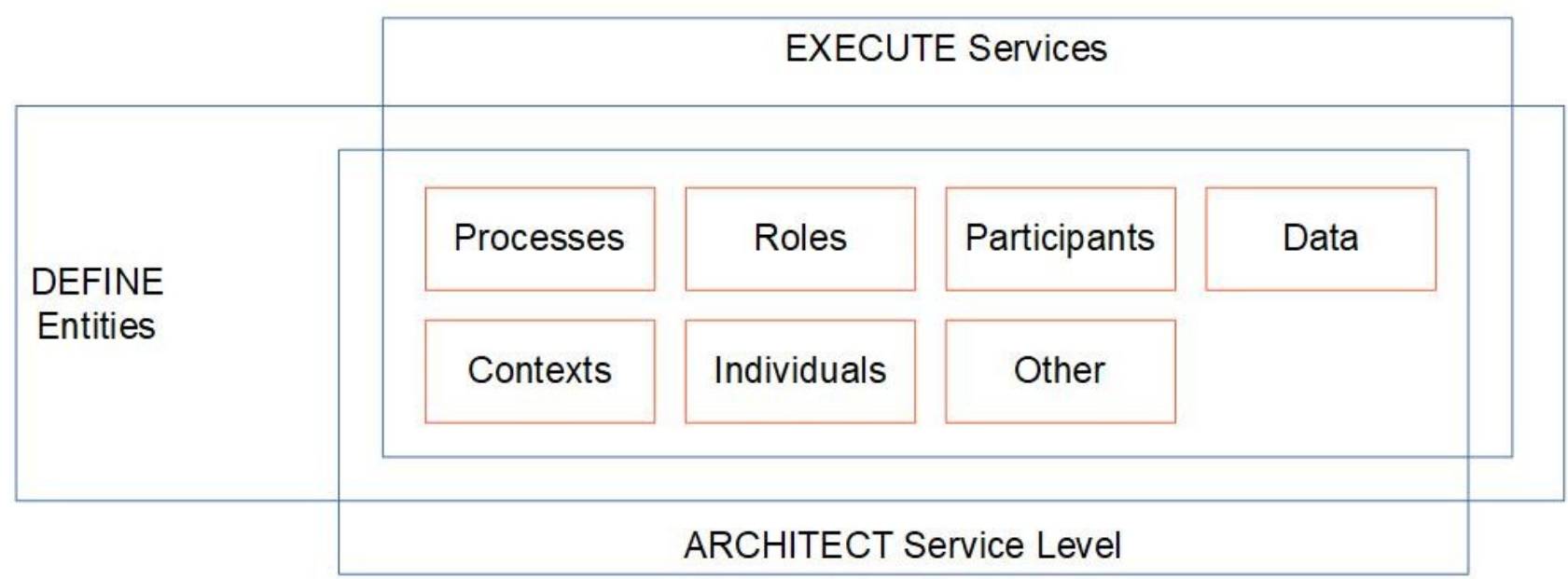

Figure 6: Some hypothesized architectural level services include the platform processes that DEFINE Entities (incl. Service Level which the services might act on), and that ARCHITECT each Service Level, as well as EXECUTE a Service. These processes are expected to be required at the infrastructure level of a GCI platform. The infrastructure domain processes and functions might be implemented in the infrastructure of a GCI platform by different services providers.

In addition to architecture level services that end users might not interact with, there is user-level functionality as shown in figure 7.

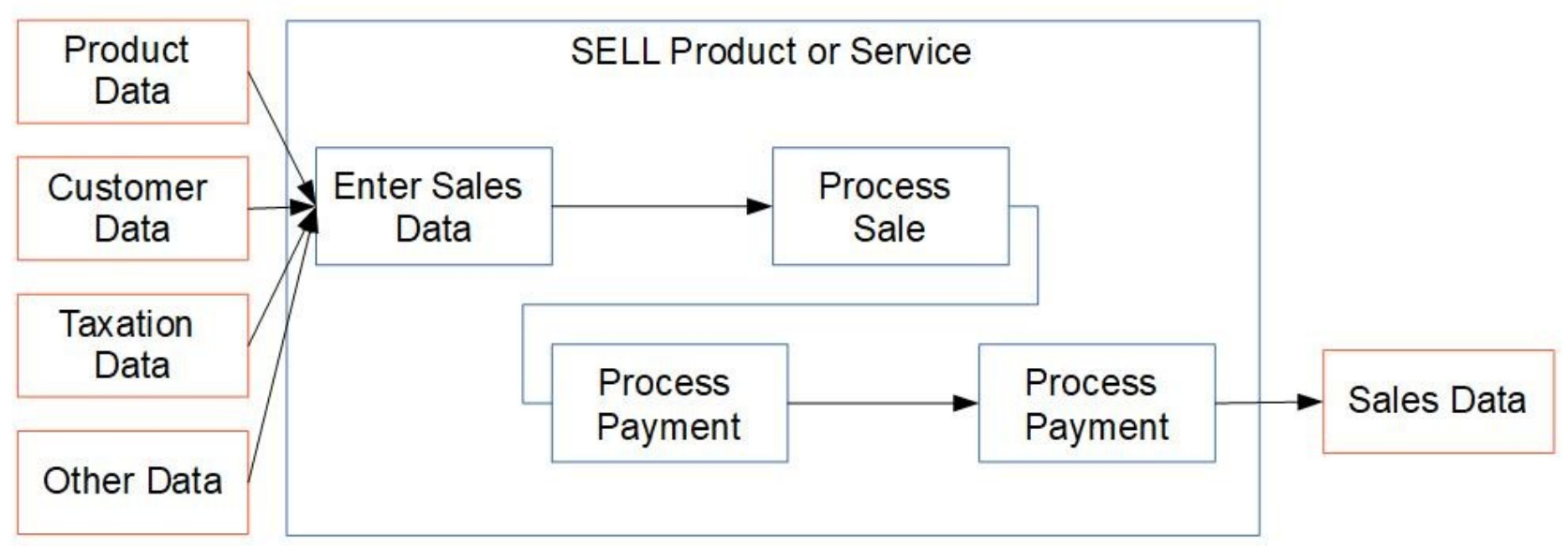

Figure 7: In addition to architecture level services, there are also user level operations. In being executed, user processes engage participants in roles that collaborate to achieve outcomes. These processes call on the platform to implement any functionality related to maintaining the Architectural Service Level.

Within a single domain, such as the Information Processing Domain, multiple sub-domains (e.g. sales, delivery) might interact.

\section{Application to Web 3.0}

Taking web 3.0 as an example of the need for functional state spaces as a domain specific modeling framework that enables the use of GCI, why should achieving a true web 3.0 require working towards 
defining functional state spaces for all required domains of web 3.0 platform behavior? Not a single web 3.0 company is currently focused in this direction. Web 3.0 development is already proceeding quite happily without what might seem like an unnecessary layer of additional complexity.

To answer this question assume that the average rate at which technology can be developed by a single company can be characterized as R, how can technology be developed with maximum cooperation by $\mathrm{N}$ companies in order to create the capacity to develop at a rate closer to $\mathrm{N}^{*} \mathrm{R}$ as opposed to being developed at maximum competition at a rate closer to $\mathrm{R}$ ? And if $\mathrm{N}$ is very large, how can the rate of development be increased through cooperation to $\mathrm{R}^{\mathrm{M}}$ so that the rate of development increases exponentially? At what rate $\mathrm{R}^{\mathrm{M}}$ does this cooperative ecosystem gain unbeatable competitive advantage over each individual company competing on their own? When companies can benefit far more by participating in an ecosystem that develops technology at a rate $\mathrm{R}^{\mathrm{M}}$ that is much greater than the rate at which any of them as an individual company can develop technology on their own, then does this cooperative development become on balance the stable reality?

These questions might be very difficult to answer on a theoretical basis, but might have very clear answers on the basis of what we experience every day in the world around us. Nowhere in the natural world do we see any collection of individual cells that is able to evolve collective functionality through competition as individual cells, where that collection of cells that each act as autonomous individual entities is able to out-compete cells that evolve collective functionality through cooperation within a single organism. This indicates that the stable balance is for all collective functionality to evolve cooperatively within an organism.

Natural evolution is an adaptive process that has been modeled as a pattern in functional state space [16]. The only known way to enable technology to evolve in this sense (as opposed to advance) is to mimic this pattern in functional state space, so that technology can be developed in self-organizing and self-sustaining way at a rate much faster than any single company can develop technology. Replicating this pattern in functional state space requires decoupling all technologies into functional components using a universal modeling framework so they can be assembled in any way required to achieve any functionality within their domain. The only universal modeling framework known today is HumanCentric Functional Modeling, which represents all of the functionality in any domain using functional state spaces.

It is further hypothesized that development of technology that is based on individual competition must eventually converge on a functional state space defined by an individual entity, while technology that is based on collective cooperation must eventually converge on a collective functional state space defined by the group. This hypothetical eventual convergence of decision-making to a single entity as a consequence of the accelerating advance of technology is called the "technology gravity well" hypothesis, which is that technology creates competitive advantage, which enables the purchase of more technology, which enables more competitive advantage, in a cycle that potentially lacks natural limits. Due to the alignment of decisions with the interests of technology owners, this implies an increasingly centralized accumulation of resources that is expected to lead to a steady centralization of technology in key areas of control. Because while median inequality might decrease, it is the inequality at the margins which is predicted to drive decision-making related to technology. Similarly, while the use of decentralized technologies might increase, it is the centralization in key areas that is predicted to drive the direction of systems. This irresistible free fall towards centralization in key areas of society due to the inevitable advance of technology has been called "the technology gravity well". This theory predicts that even movements focused on decentralization such as web 3.0 will be centralized in hidden ways that tie technology to a single vendor, and that this centralization will be introduced at an 
increasing rate and level of complexity that can't easily be detected much less addressed by any policy. A General Collective Intelligence however is predicted to enable a true web 3.0 at any pace of development by defining decentralization as an objective pattern of stability that can be maintained at any speed and scale of activity.

Assuming that as predicted the collective functional state space can be far larger than any individual functional state space, this suggests that without collective development some solutions will not be reliably within that functional state space, and therefore that without collective development some problems cannot be reliably solved. This "solvability of classes of group problems" hypothesis [17], is that there are classes of problems that it is believed cannot reliably be solved today due to misalignment between outcomes that are optimal for the group and outcomes that are optimal for the individual or subset of individuals choosing the solution when decision-making is performed in a centralized way. This centralized decision-making, and these resulting problems can occur in any process along the entire life-cycle of web technology from research, design, development, sales, or delivery, to administration and maintenance, or retirement at the end of that life-cycle. Many web technologies, particularly blockchain or web 3.0, are decentralized in some aspects, but lack decentralization in others such as in administration of the development process.

Furthermore, not only can different types of problems be solved, but the complexity of problems that can be solved is predicted to be significantly higher in a larger and denser functional state space, since complexity is defined by distance through functional state space as well as density. This "wicked problems" hypothesis [18] is that a wicked problem for an individual is one that is defined by a longer path through a greater density of concepts in conceptual space than an individual cognition can reliably reason through, and that a wicked problem for a group without a collective cognition is one that is defined by a longer path through a greater density of concepts in the conceptual spaces of the individuals, than they can reliably reason through. A wicked problem for a collective cognition is one that is defined by a longer path through a greater density of concepts in the collective conceptual space, than the group can reliably reason through. Since a collective cognition is predicted to have the potential capacity for exponentially greater intelligence, what is a wicked problem for an individual might not be a wicked problem for a GCI.

In web 3.0 application or platforms, Human-Centric Functional Modeling aims to create the capacity to decouple all application or platform functionality into a library of functional components spread across a number of functional domains. This creates the opportunity to assemble software applications or platforms using whatever set of functional components that is optimal for a given problem. Assuming that this assembly can be achieved through a system such as General Collective Intelligence that is predicted to exponentially increase the general problem-solving ability of groups, this in turn creates the possibility of exponentially increasing the speed and scale of development.

This different from current development in that when a set of operations can be defined that "span" the entire functional state space, then all possible behaviors of software in that domain can be represented in terms of some composition of those operations. In the case of a hypothetical blockchain domain for example, this suggests that all blockchain platforms could be decoupled into a library of functional components, and that a system with general problem-solving ability such as a GCI might be used to dynamically select whatever set of components is needed to execute any functionality in that domain in a way that is optimal for the group. Taken together, these properties suggest that such a platform might have exponentially greater capacity to solve all possible web 3.0 problems.

\section{Discussion}


Various surveys of techniques in model driven architecture found a number of shortcoming. One author stated: "while practitioners experience benefits of modelling for analysis and design, requirements engineering, quality management, implementation and deployment, they still struggle with external tool integration/model transformation and export, cognitive fit, visual expressiveness, high effort required in acquiring skills, automated analysis and high effort required in using tools" [9]. Another author cited the lack of standardization due to the complexity of modeling heterogeneous systems, or perhaps the complexity due to lack of standardization [10]. Even in problems of restricted scope such as the blockchain [11], or networking [12], these issues remain a factor [13]. With all these difficulties it's not surprising that the resulting lack of completeness and consistency has prevented convergence among the various techniques, and as a result a coherent meta-model of the clusters into which collaborative and other model-driven engineering efforts might be classified has not yet emerged [14].

How might defining functional state spaces lead to an exponential increase in the deployment of domain specific modeling and domain specific modeling tools if managing even the current modest deployments is difficult? Assume that all functionality of a system in a given domain of its behavior can be understood in terms of $\mathrm{N}$ functional states. The number of all possible behaviors of the system within that domain are defined by all the possible paths between those $\mathrm{N}$ states.

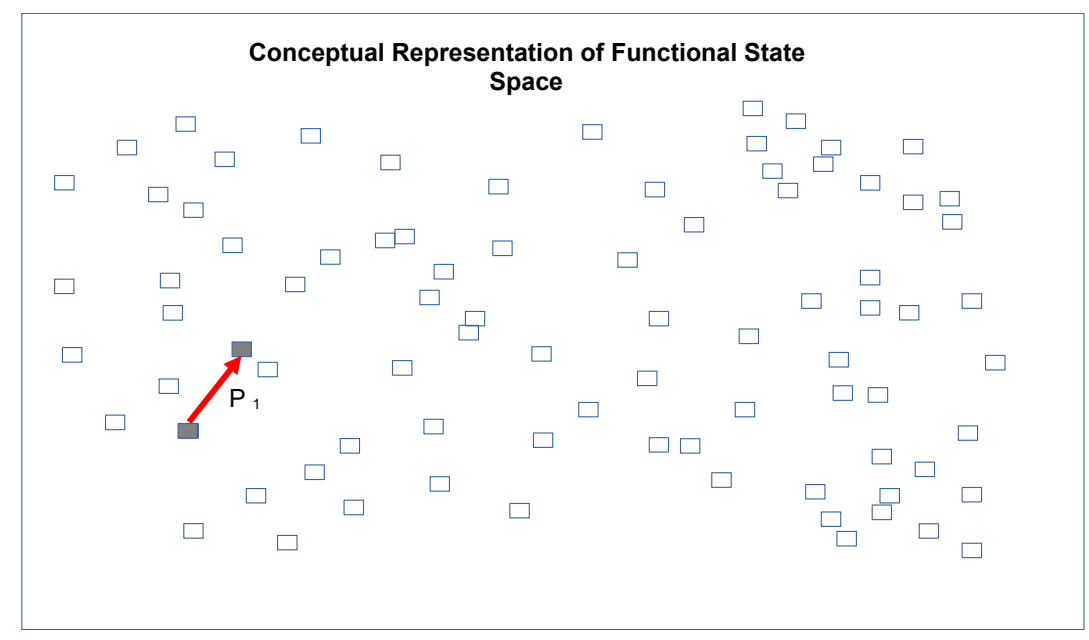

Figure 8: Every path $P_{i}$ in functional state space is an operation that can potentially be represented in a domain specific modeling tool.

Since each state can potentially transition to $\mathrm{N}-1$ other states, then there are $\mathrm{N}(\mathrm{N}-1)$ direct or first order transitions. Assuming that a transition does not end up at the same state at which it began, there are $\mathrm{N}(\mathrm{N}-1)(\mathrm{N}-2)$ second order transitions. Assuming that the maximum order of behavior is $\mathrm{M}$, then represent the total number of possible behaviors up to order $\mathrm{M}$ for a system that has $\mathrm{N}$ functional states as the total $\mathrm{T}$.

$$
T=\sum_{i=1}^{i=M}(N-i) !
$$

From Stirling's approximation, this number can be exponentially greater than N. Currently, any domain specific modeling tool can be used to represent some subset of behavior of a system within a given domain, but how do the number of those domain specific models and the number of opportunities to deploy those models compare to the number of opportunities to deploy domain specific modeling that 
might be created in the future through the functional state space approach? For simplicity, assume that on average each domain specific model contains a subset of $\mathrm{X}$ behaviors, and assume that there are $\mathrm{Y}$ models. Then if there are $\mathrm{Z}$ applications for domain specific modeling, there might be as few as 1 opportunity to deploy a domain specific modeling tool if all those models overlap (all the models describe the same subset of behavior but perhaps in terms of a different set of operations so that each model competes with each other). This tool deployment then involves modeling each of X operations once. On the other hand, if every model describes behavior that only overlaps moderately, there might be as many as $\mathrm{T} / \mathrm{Y}$ possible opportunities to deploy domain specific modeling, with the actual number of opportunities limited by Z, each of which tool deployments again involves modeling X operations. However, as the number of domains increases, avoiding overlap while developing the models independently becomes increasingly difficult. Furthermore, as the number of operations being modeled grows, making use of those models becomes increasingly difficult as well. If there is no overlap whatsoever (all behavior is modeled as being part of a contiguous functional state space), there might be as many as some unimaginably large number $T$ possible operations that might be modeled using domain specific modeling. Furthermore the search of operations by ontology permitted by initial approximations of a General Collective Intelligence is predicted to make a far larger number of operations manageable, and the eventual semantic search predicted to be made possible by a true General Collective Intelligence, has the potential to make even an exponentially greater number of operations manageable. Furthermore, collective development orchestrated by a General Collective Intelligence is predicted to exponentially increase the functional state space, and therefore to increase the number of applications Z. Under Stirling's approximation, this increase in the number of potential applications of domain specific modeling is also expected to be exponential.

However, this discussion of some number $\mathrm{N}$ of functional states doesn't convey an understanding of the vastness of conceptual space, which is important in understanding how domain specific modeling might optimally be used within any subset of it. To understand that vastness, consider the number of words in the average vocabulary to be an approximation for the number of concepts in each individual's conceptual space. An average adult English speaker actively uses around 20,000 words [8], and can passively comprehend around 40,000 words. Understanding the openness of the total possible individual conceptual space as well as the potentially exponentially greater volume that the collective conceptual space might occupy is less intuitive. Aside from the words actively used or passively understood by a given individual, there are also the words that exist in their language. It might not be possible to count the words in a language since there is no way to tell whether a word is missing, but it is certainly possible to count the words in a dictionary, though such totals don't take account of entries with senses for different word classes (such as noun and adjective) and homographs. The number of words in some modern dictionaries ranges from over $1 \mathrm{M}$ for the Korean online dictionary, to tens of thousands for various other languages. Assuming that each word represents a concept, assuming that there is only a single instance of the entity represented by that concept, and assuming that there are the same number of concepts in every language, then at minimum there are over $1 \mathrm{M}$ concepts that can occupy an individual's conceptual space. However, for each word representing a concept, like "elephant", there might be billions of elephants that currently exist, that have existed at some point during the history of the earth, or that will exist in any real or imaginary world, all of which might be described by that concept. Therefore elephant is not just a single point in conceptual space, its a region in conceptual space that potentially contains billions of points. A single individual might not be aware of all of these concepts of elephants, and therefore these points might not be within their individual conceptual space, but if these concepts exist across all of humanity, then they exist within the collective conceptual space. Given that there are nearly 8 billion people on earth, we can see how it is possible that the collective conceptual space might be a billion times larger than the conceptual space of any individual, even if many of the concepts in each individual's conceptual space overlap. 
As for defining a finite ontology of operations in a domain specific model which might be implemented by software processes in order to navigate between a manageable subset of this potentially vast number of concepts, all software can be considered as an automation of human reasoning processes. Reasoning processes consist of either type 1 (intuitive) reasoning that is represented as a direct path between two concepts in conceptual space, or type 2 (rational methodical) reasoning which is represented as a path through intermediate concepts constituting a chain of logic. Any machine learning program can be represented as an automation of a type 1 reasoning process, and the logic of any procedural software program can be represented as an automation of a type 2 reasoning process. Therefore all software programs can be mapped to some path in conceptual space. However, in order to be able to construct all such software programs with such a domain specific model, we must have the capacity to construct any path through conceptual space. In the domain of concepts the four operations hypothesized to "span" all conceptual space are believed to make this possible. However, these operations are too abstract to be easily translated to language by humans without the assistance of computer automation.

The relationship between domain specific modeling and functional state space is then that the operations in the ontology of each domain specific language represent some subset of the potentially vast number of paths though the functional state space corresponding to that domain. As an example, taking a domain like the "Information Processing Domain", or the "Visual Processing Domain", our choices remain within this same range of defining an ontology for the domain using a minimal set of functions that "span" all possible paths through that functional state space but are very abstract, and defining an ontology for the domain using some subset of the possible reasoning paths within that domain that are in common usage. Which approach we take might vary depending on the context. For example, in the case of the potentially thousands of blockchain platforms, each of which is difficult to understand and therefore lack any intuitive representation that is in common usage, it's hypothesized that defining a set of four basic abstract operations that span all blockchain platforms might finally enable the creation of a "cognitive blockchain" platform that provides complete blockchain interoperability. For blockchain platforms, the high level of abstraction in these functions might not be an impediment because there is no common intuitive understanding in any case. In the case of user interfaces on the other hand, the "Visual Processing Domain" defines a few dozen operations that are hypothesized to intuitively represent the vast majority of user interfaces. These operations might not span all possible user interfaces, but might still be very useful.

When a given set of operations is defined within a domain such as the "Visual Processing Domain", another competing set of operations might come to be proposed within that same domain. It is expected to be possible for a user interface model defined in one representation of the visual processing domain to be translated to another representation of the visual processing domain, though this remains to be confirmed in practice. The point is to emphasize the importance of providing a path between each representation and each other representation, so that groups can evolve from one to the other according to the relative fitness of each representation, in order to achieve consistency and convergence.

\section{Conclusion}

According to the theory of Human-Centric Functional Modeling, defining a functional state space capable of providing a complete representation of the behavior of any given system in a given domain requires defining a minimal set of the most basic possible behaviors with which all other behaviors might be composed. The motivation to engage in this difficult exercise is that all open functional state spaces are hypothesized to be spanned by some combination of only four operations. This is predicted to be true for the functional state space that describes cognition, the functional state space that describes the physical universe, or any other open functional state space. 
Domain specific modeling in general suffers from the brittleness that comes with each individual, business, government, or other entity defining their own ontology of behaviors, as opposed to defining a framework in which groups can reliably come to a consensus regarding the complete set of behaviors that any system is capable of. Functional state spaces for this reason provide a framework that can potentially be used to define not just one domain specific modeling language, but a framework that can be used to define a domain specific modeling language for each domain, and for all possible domains and the relationships between them so that all behavior in all possible domains is represented.

This paper has provided a brief conceptual overview of how the emerging science of Human-Centric Functional Modeling or HCFM might be used to define functional state spaces that might serve as a domain specific modeling framework for General Collective Intelligence or GCI platforms. The purpose of this paper was not to delve into any of these modeling domains or their implementations in detail, each of which might occupy a book on its own. Instead the purpose was merely to introduce the concept that a system of such domains might form the basis with which to construct a General Collective Intelligence platform, and that such a platform might radically increase the collective impact that is achievable through domain specific modeling tools, and in addition might make some outcomes (such as the complete implementation of web 3.0 or complete blockchain platform interoperability) reliably achievable where this has never been the case before.

\section{References}

[1] Williams, A. E. Defining a Continuum from Individual, to Swarm, to Collective Intelligence, to General Collective Intelligence, International Journal of Collaborative Intelligence, in press (2022) [2] Williams, A. E. Approximating an Artificial General Intelligence or a General Collective Intelligence, International Journal of Collaborative Intelligence, in press (2022)

[3] Beigi, S., \& Heylighen, F. (Accepted/In press). Collective Consciousness Supported by the Web: healthy or toxic? In Computational Collective Intelligence: 13th International Conference, ICCCI 2021, Proceedings (LNAI). Springer.

[4] Suppe, Frederick. The semantic conception of theories and scientific realism. University of Illinois Press, 1989.

[5] Lloyd, Elisabeth A. "A semantic approach to the structure of population genetics." Philosophy of Science 51.2 (1984): 242-264.

[6] Niiniluoto I. (1987) Quantities, State Spaces, and Laws. In: Truthlikeness. Synthese Library (Studies in Epistemology, Logic, Methodology, and Philosophy of Science), vol 185. Springer, Dordrecht. https://doi.org/10.1007/978-94-009-3739-0_3

[7] Williams, A. E. (2020, September). A model for artificial general intelligence. In International Conference on Artificial General Intelligence (pp. 357-369). Springer, Cham.

[8] Brysbaert M, Stevens M, Mandera P and Keuleers E (2016) How Many Words Do We Know? Practical Estimates of Vocabulary Size Dependent on Word Definition, the Degree of Language Input and the Participant's Age. Front. Psychol. 7:1116. doi: 10.3389/fpsyg.2016.01116 [9] Verbruggen, Charlotte, and Monique Snoeck. "Model-Driven Engineering: A State of Affairs and Research Agenda." Enterprise, Business-Process and Information Systems Modeling. Springer, Cham, 2021. 335-349.

[10] Mohamed, Mustafa Abshir, Geylani Kardas, and Moharram Challenger. "Model-Driven Engineering Tools and Languages for Cyber-Physical Systems-A Systematic Literature Review." IEEE Access 9 (2021): 48605-48630.

[11] Levasseur, Olivier, Mubashar Iqbal, and Raimundas Matulevičius. "Survey of Model-Driven Engineering Techniques for Blockchain-Based Applications." Proceedings http://ceur-ws. org ISSN 1613 (2021): 0073. 
[12] Hammoud, Adra, et al. "The contribution of mda in software-defined network: A survey."

Advancements in model-driven architecture in software engineering. IGI Global, 2021. 118-134.

[13] Barangi, Hamed, et al. "Model-Driven Software Engineering: A Bibliometric Analysis." Journal of Computing and Security 8.1 (2021): 93-108.

[14] David, Istvan, et al. "Collaborative model-driven software engineering: a systematic update." 2021

ACM/IEEE 24th International Conference on Model Driven Engineering Languages and Systems

(MODELS). IEEE, 2021.

[15] Williams, A. E. (2022, March 12). Selecting Between Semantic Modeling of Intelligence and Semantic Modeling of Systems. https://doi.org/10.31730/osf.io/gdn5y

[16] Williams, A. E. (2021, June 13). General Problem-Solving Ability in Natural Systems as a Model for Computation. https://doi.org/10.31730/osf.io/5x3g7

[17] Williams, A. E. (2022, March 2). Using General Collective Intelligence to Re-think DecisionSystems and Other Information Technologies \& Information Systems to Better Inform Pandemic Responses. https://doi.org/10.31730/osf.io/q7kvd

[18] Williams, A. E. (2022, February 7). Is Systems Complexity a Property in a Functional State Space of Each System?. https://doi.org/10.31730/osf.io/rnysw 